\title{
A SIMULATION-BASED APPROACH TO INVENTORY MANAGEMENT IN BATCH PROCESS WITH FLEXIBLE RECIPES
}

\author{
Long $\mathrm{He}$ \\ Industrial Engineering and Operations Research \\ University of California, Berkeley \\ Berkeley, CA 94704, USA
}

\author{
Simin Huang \\ Industrial Engineering \\ Tsinghua University \\ Beijing 100084, CHINA
}

\author{
Zuo-Jun Max Shen \\ Industrial Engineering and Operations Research \\ University of California, Berkeley \\ Berkeley, CA 94704, USA
}

\begin{abstract}
Batch processes are widely adopted in many manufacturing systems with raw materials from mining or agricultural industries. Due to variations in both raw material quality and market conditions, variations in the recipes are used in production. Such recipe flexibility is not on design but on the operation that allows adjustments of recipe items aiming to achieve better performance than traditionally fixed recipes. In this paper, we study the inventory investment, recipe selection and resource allocation decisions in batch process systems with flexible recipes. A two-stage stochastic mixed integer program formulation is developed for each period. Moreover, the system updates its inventory investment decisions based on new demand data from previous periods by a simulation-based approach. Benefits of implementing flexible recipes over traditional fixed recipes are investigated in the numerical studies.
\end{abstract}

\section{INTRODUCTION}

Oil consumption has been escalating in the past decades, especially in emerging economies regions, such as Asia. Meanwhile, the dramatically increasing oil price is impeding the growth of the world economy. Despite its increasing trend, oil price also exhibits high volatility. After it reached the record peak US\$ 145 in July 2008, it fell significantly to US\$ 30.28 a barrel on December 23, 2008. Such increasing trend together with jumps of prices also prevails in other commodities over the past decades as shown in Figure 1. This phenomenon leads to higher manufacturing costs as well as more difficulties in supply chain management under price uncertainty among many industries.

Facing such challenges, joint inventory investment and allocation decision making becomes an important tool that makes the manufacturing systems robust. Consider an oil refinery that converts crude oil into profitable petroleum products such as gasoline, diesel, kerosene, heating oil and asphalt. Those products are actually inputs for further manufacturing processes. Generally, it operates in 3 phases: crude oil unloading and blending, fractionation and reaction processes and product blending and shipping. In the first phase, crude oil of different grades is transported by crude oil marine vessels from different regions. Since the properties of crude oil highly depend on its origins, there are usually dedicated storage tanks for crude oil of different grades. In many situations, before crude oil enters distillation, the first step of production, 


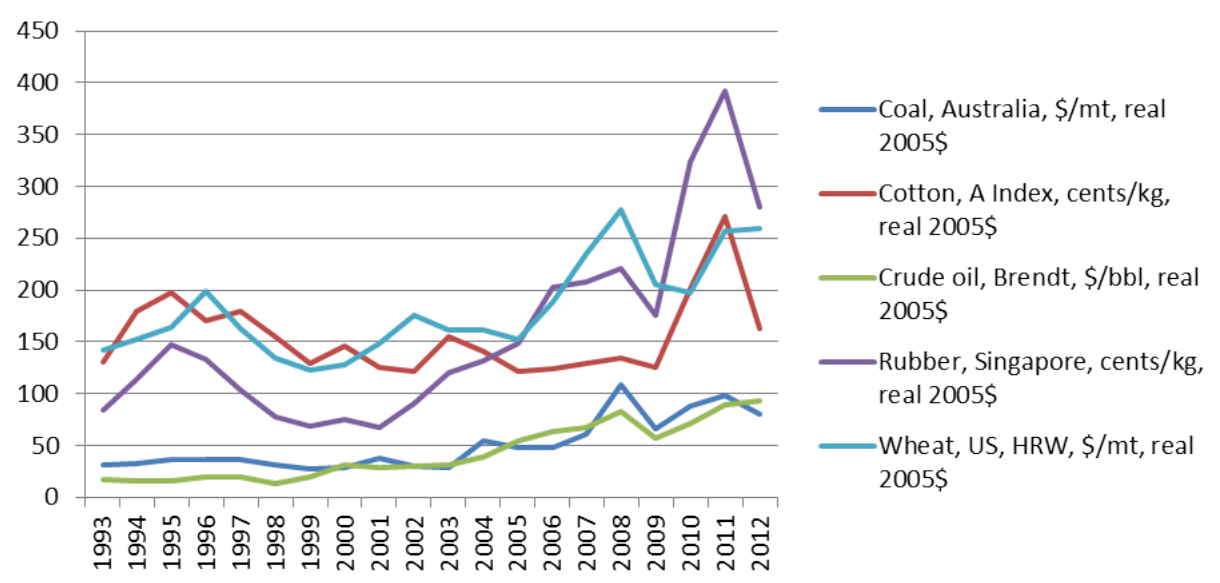

Figure 1: Selected Commodity Prices in Past 20 Years.

different grades are blended to achieve certain properties, such as viscosity and density, in order to meet the production requirements.

The manufacturing process presented above belongs to batch process that primarily schedules short production runs of products (Connor 1986). Batch process industries often obtain their raw materials from mining or from agricultural industries. These raw materials have natural variations in quality (Rutten and Bertrand 1998). Some common batch processes can be found in fields such as oil refining, agricultural, chemicals and fertilizers. (Schuster and Allen 1998) illustrates how Welch's Inc manages grape-processing among plants using linear program models. In that case, grapes are usually processed in plants located near growing areas. To maintain national consistency, Welch's often transfers juice for blending between plants. The selection of recipes is a key decision that affects the profitability via both operational costs and production capacity. The nature of variations in both raw material quality and market conditions often lead to the variations in the recipes. Such recipe flexibility is not on design but on the operation that allows adjustments of recipe items aiming to achieve better performance than traditionally fixed recipes. Here, flexible recipe refers mainly to the adjustments of recipe items as input of batch process in response to market conditions, i.e. demand arrivals.

In this paper, we simplify the system by considering three types of goods: raw materials, ingredients and final products. In the grape-processing case, we regard grapes from different growing areas as raw materials, intermediate juice of different concentration as ingredients and packaged juice on market as final products. The batch process is simplified into two phases: separation and blending. Since different raw material grades have various concentration of desired ingredients, in the separation stage, those ingredients are separated first from raw materials. Then a combination of ingredients are blended into final products that meet certain specifications.

The remainder of this paper is organized as follows: We begin in $\S 2$ by reviewing related literature. In $\S 3$, an application of flexible recipes is illustrated by a simple case. A two-stage stochastic mixed integer program for the basic model is formulated in $\S 4$. We then discuss a simulation-based approach to the proposed model in $\S 5$. In $\S 6$, we conduct a numerical study to assess the performance of the simulation-based approach and benefits of flexible recipes over fixed recipes. Finally, concluding remarks are presented in $\S 7$.

\section{LITERATURE}

There are mainly two streams of literature related to our work. In the first stream, given the structure of the system, optimal investment decisions are analyzed under demand uncertainty and/or inventory procurement cost variability. (Fine and Freund 1990) investigate the optimal capacity investment problem in single 


\section{He, Huang, and Shen}

period by two-stage stochastic programming with discrete demand distribution. Their study focuses on the case with two products, two dedicated resources, one flexible resource. While in our study, we allow the demand distribution to be unknown and the system handles multiple raw materials and final products. Following the lead of (Fine and Freund 1990), under a two-product firm, (Van Mieghem 1998) analyze the optimal investment in flexible resources as a function of margins, costs and multivariate demand uncertainty. Contrary to the previous work, they show that it can be advantageous to invest in flexible resource even with perfectly positively correlated product demands. (Harrison and Van Mieghem 1999) study the optimal investment strategy with a multi-dimensional newsvendor model and conclude a critical fractile property for the optimal investment levels. Given the structure of an assemble-to-order system, (Akçay and Xu 2004) formulate the join inventory replenishment and component allocation problem into a two-stage stochastic program and propose an order-based component allocation rule for the second stage problem.

In the second stream, the applications of flexible recipes in batch processes are mostly studied. (Rutten and Bertrand 1998) study the balancing of safety stock costs and recipe flexibility costs for batch industries with high customer service requirements. They conclude that under certain circumstances the use of recipe flexibility can lead to lower costs when compared to using fixed recipes. (Keesman 1993) investigates the application of flexible recipes for batch process optimization and applies an adaptive feedforward control strategy for a priori known disturbances in the process inputs. Furthermore, a new framework that fully exploits the inner flexibility of batch processes at the plant level is developed by (Romero et al. 2003). Their framework considers a batch recipe model that interacts with a plant-wide model to constitute the flexible recipe model. The most related work to ours, under batch process manufacturing, is done by (Karmarkar and Rajaram 2001). They formulate the grade selection and blending problem as a nonlinear mixed-integer program with fixed cost for grade selection and inventory holding cost. However, they assume the annual demand is known and constant for each final products.

Our work is different from the literature mainly in two ways. First, in our model, the recipe flexibility is embedded in the operations of batch processes, rather than the system design as seen in literature on process flexibility. Second, we study the decisions of inventory investment, recipe selection and resource allocation in an integrated model.

\section{EXAMPLE OF FLEXIBLE RECIPE APPLICATION}

In this section, we briefly illustrate the application of flexible recipes in batch process. Consider a manufacturer, i.e. refinery or food processing factory, whose operations can be categorized as separation and blending stages. There are 3 final products made from 3 raw materials. The raw material inventory is given as $Z=\left(z_{1}=200, z_{2}=300, z_{3}=400\right)$ units, where $z_{i}$ is the inventory of raw material $i$. The raw material cost is $C=\left(c_{1}=6, c_{2}=4, c_{3}=3\right)$ dollars per unit, where $c_{i}$ is the purchase cost of raw material $i$ per unit. In the batch process, raw materials are separated first into 3 ingredients, depending on their concentration in raw materials. The ingredient concentration matrix for raw materials is

$$
P=\left(\begin{array}{lll}
0.6 & 0.3 & 0.1 \\
0.4 & 0.4 & 0.2 \\
0.3 & 0.4 & 0.3
\end{array}\right)
$$

where row $i$ represents raw material $i$ and column $j$ represents ingredient $j$. The element on row $i$ and column $j$, denoted by $p_{i j}$, is the proportion of ingredient $j$ contained in a unit of raw material $i$. Then in the blending stage, final products are blended from those ingredients. The ingredient requirement matrix for final products is

$$
A=\left(\begin{array}{lll}
0.8 & 0.2 & 0 \\
0.7 & 0.2 & 0.1 \\
0.6 & 0.3 & 0.1
\end{array}\right)
$$

where row $k$ represents final product $k$ and column $j$ represents ingredient $j$. The element on row $k$ and column $j$, denoted by $\alpha_{k j}$, is the proportion of ingredient $j$ required in a unit of final product $k$. 


\section{He, Huang, and Shen}

In a system that implements fixed recipes, it determines the optimal fixed recipe before any demand arrivals. The resulting optimal fixed recipe in this case is:

$$
B=\left(\begin{array}{lll}
0.53 & 0.44 & 1.03 \\
0.40 & 0.18 & 1.29 \\
0.32 & 0.11 & 1.22
\end{array}\right)
$$

where row $k$ represents final product $k$ and column $i$ represents raw material $i$. This matrix is similar to BOM. That is, the element on raw $k$ and column $i$, denoted by $b_{k i}$, is the amount of raw material $i$ required in a unit of final product $k$.

For simplicity, we assume that the demand for each final product follows Bernoulli distribution with 0.5 probability equals 100 units and 0.5 probability equals 200 units. When the system sees demand arrivals, it determines the optimal flexible recipes that maximize its revenue with $R=\left(r_{1}=10, r_{2}=8, r_{3}=6\right)$ dollars, where $r_{k}$ represents the revenue of a unit final product $k$.

Table 1 summarizes the computational results of expected profit and shows that the flexible recipes enable

Table 1: Expected Profit Summary (in dollars)

\begin{tabular}{c|ccc}
\hline & Flexible Recipes $(R 1)$ & Fixed Recipes $(R 2)$ & Improvements $=\frac{R 1-R 2}{R 2}$ \\
\hline Current Setting & 3525 & 2904.6 & $21.36 \%$ \\
Demand $\times 2$ & 4350 & 3199.8 & $35.95 \%$ \\
Demand $\times 0.8$ & 2880 & 2643.7 & $8.94 \%$ \\
\hline
\end{tabular}

the system to achieve higher profit via better resource utilization, especially under the presence of large demand variance.

\section{THE BASIC MODEL}

As shown in Figure 2, the batch process consists of two stages: in the separation stage, raw materials are processed into a set of ingredients; in the blending stage, a selection of ingredients are blended into final products to fulfill the demands. In the oil refinery industry, for instance, the raw materials are different crude oil grades. The three most quoted oil grades are North America's West Texas Intermediate crude (WTI), North Sea Brent Crude, and the UAE Dubai Crude. Depending on the mixture of hydrocarbon molecules, crude oil varies in color, composition and consistency. Different oil-producing areas yield significantly different varieties of crude oil. The ingredients are the intermediate products such as light ends, naphtha, kerosene, distillate, atmospheric residua, vacuum gas oil and vacuum residua. Final products are various gasoline types, lubricants, petrochemicals, diesel, asphalt, etc,.

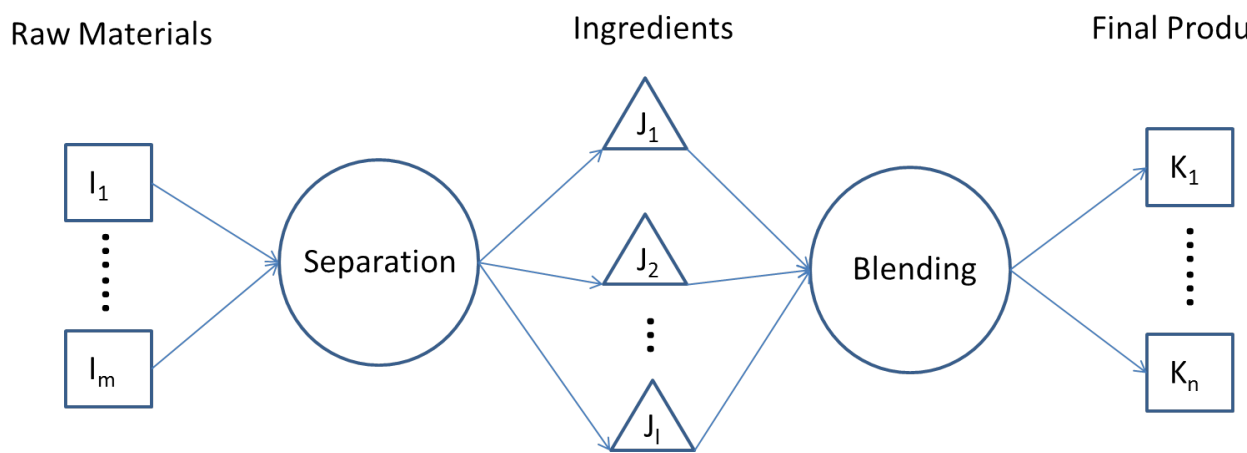

Figure 2: Simplified Batch Process

Our model is to determine the inventory level at the beginning of each period before any demand arrivals. We assume all leftover inventory by the end of each period is disposed because of time sensitivity of raw materials, i.e. perishability in grape-processing. As a result, at the beginning of each period t, we 
only need to solve a subproblem of single period planning with available historical data up to period t. Therefore, we focus on solving a single period problem.

There are basically three types of costs: raw material procurement cost, grade selection cost and final product revenue. Similar to the definition by (Karmarkar and Rajaram 2001), the grade selection cost incurs when a grade is selected by a recipe into separation stage and the cost varies by grades. Raw material procurement cost can be the real option or spot market price. The model we propose is able to optimize investment on raw materials together with flexible recipe selection for production when the system sees demands. It can be easily extended to the systems with many separation and blending stages in serial structure.

The event sequence is illustrated in Figure 3. At the beginning of period t, the system first invests in raw material inventory with total procurement $\operatorname{cost} \sum_{i} c_{i} z_{i}$, where $c_{i}$ is the unit cost of raw material $i$ and $z_{i}$ is the amount of raw material $i$ purchased. After the system sees the demand arrivals $D=\left(d_{k}\right)$, where $d_{k}$ is the demand of final product $k$, it then selects the optimal recipe to satisfy the demands. Under the presence of grade selection cost, it is not always profitable to satisfy as much demand as possible. As discussed above, the recipe selection cost is the sum of grade selection costs, given by $\sum_{i} f_{i} y_{i}$, where $f_{i}$ is the grade selection cost of raw material $i$ and $y_{i}$ is the selection decision of raw material $i$. Lastly, the fulfillment of demands generates total revenue $\sum_{k} r_{k} x_{k}$, where $r_{k}$ is the unit revenue of final product $k$ and $x_{k}$ is the fulfilled demand of final product $k$. We assume that material transformation processes are linear. Another assumption is that the material loss in separation and blending stages are negligible. Indeed, if the loss is consistent and fractional in the process, we can introduce some discount factors in the formulation so that the structure of the model is still preserved.

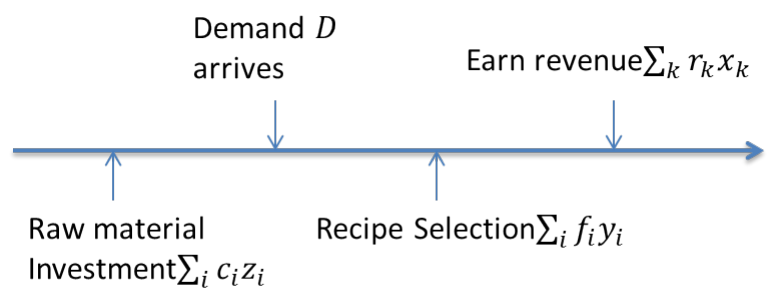

Figure 3: Event Sequence Diagram in Period $t$

The decision making process for the system with flexible recipes differentiates itself from the newsvendor model with the postponement in final product blending. In the newsvendor model, the final products are produced ahead and the total manufacturing cost can be assessed before demand arrivals. As a result, in the newsvendor model, the critical fractiles and subsequently the raw material inventory levels can be determined in advance. While in a batch process with flexible recipes, the system makes tradeoffs between revenue and recipe selection cost after demand arrivals.

We formulate the basic model as a two-stage stochastic mixed-integer program:

$$
\Pi=\max \mathbb{E}_{D} \pi(Z, D)-\sum_{i \in I} c_{i} z_{i}
$$


where

$$
\begin{aligned}
\pi(Z, D)= & \max \sum_{k \in K} r_{k} x_{k}-\sum_{i \in I} f_{i} y_{i} \\
\text { s.t. } & \sum_{k \in K} \alpha_{k j} x_{k} \leq \sum_{i \in I} p_{i j} z_{i} y_{i}, \forall j \in J \\
& x_{k} \leq d_{k}, \forall k \in K \\
& y_{i} \in\{0,1\}, \forall i \in I
\end{aligned}
$$

Uppercase is for vectors while lowercase is for scalars. The first stage of the formulation, equation (1), maximizes the expected total profit of the system. Given raw material inventory level vector $Z=\left(z_{1}, z_{2}, \ldots, z_{I}\right)$ and demand arrival vector $D=\left(d_{1}, d_{2}, \ldots, d_{K}\right)$, the second stage with recourse maximizes as in equation (2) the total revenue minus total grade inclusion cost. The constraint (3) states that the supply of each ingredient $\sum_{i \in I} p_{i j} z_{i} y_{i}$ must be no less than the ingredient requirement for producing $X$ amount final products which is $\sum_{k \in K} \alpha_{k j} x_{k}$. Here $\alpha_{k j}$ is the amount of ingredient $j$ required to produce a unit of final product $k$ and $p_{i j}$ is the amount of ingredient $j$ contained in a unit of raw material $i$. Because the initial inventory investment is sunk cost, $\left(z_{1} y_{1}, z_{2} y_{2}, \ldots, z_{I} y_{I}\right)$ shows that the system chooses to use up all selected raw materials. Constraint (4) represents that demand fulfillment can not exceed the demand arrivals. The recipe selection is determined by the binary decision vector $Y$ expressed in constraint (5).

\section{SIMULATION-BASED OPTIMIZATION}

Our solution approach to the proposed two-stage stochastic mixed-integer program consists of two modules: demand simulator and SAA optimizer, as shown in Figure 4. Given the available historical demand data, the demand simulator generates simulated demand arrivals for period $t$. There are several ways to simulate or forecast demands based on previous information. Here, we use Bootstrap sampling in the demand simulator.

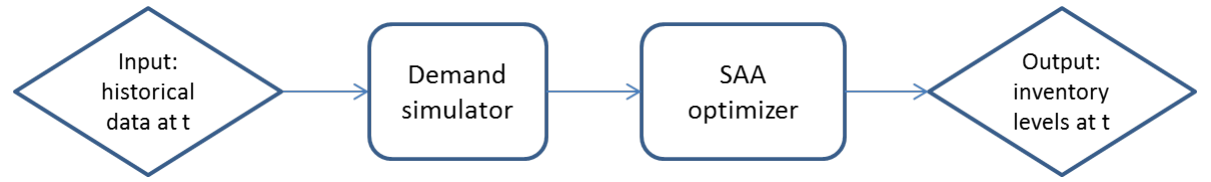

Figure 4: Solution Approach

With the simulated demand arrivals, the second module finds the optimal inventory levels by Sample Average Approximation (SAA), an simulation-based approach. The algorithm is modified from the SAA method provided in (Akçay and Xu 2004). A detailed introduction of the SAA method can be found in (Shapiro and Homem-de Mello 1998). Let $\Pi\left(Z^{*}\right)$ be the optimal solution to the two-stage stochastic program in the basic model (1)-(5) and $Z^{*}$ be the associated optimal inventory levels. We start with generating $M$ independent samples of random vector $\mathbf{D}$ from the demand simulator, each of size $N$. That is, $\mathbf{D}^{l}=\left(D^{l, 1}, D^{l, 2}, \ldots, D^{l, N}\right)$ is the realization of the $l$ th sample, where $D^{l, h}=\left(d_{1}^{l, h}, d_{2}^{l, h}, \ldots, d_{K}^{l, h}\right)$ with $d_{k}^{l, h}$ as the realization of demand for final product $k$ in the $h$ th vector of the $l$ th sample realization. We solve the SAA problem referring to each sample, as below:

$$
\begin{aligned}
& \max _{Z^{l}}\left\{\Pi^{N}\left(Z^{l}\right)=\frac{1}{N} \sum_{h=1}^{N} \pi\left(Z^{l}, D^{l, h}\right)-\sum_{i \in I} c_{i} z_{i}^{l}\right\} \\
& \text { s.t. } \\
& \quad \text { Constraints }(3)-(5) \text { for each } D^{l, h}, h=1, \ldots, N
\end{aligned}
$$


where $Z^{l}=\left(z_{1}^{l}, z_{2}^{l}, \ldots, z_{I}^{l}\right)$

For $l=1, \ldots, M$, let $\Pi^{N}\left(\hat{Z}^{l}\right)$ be the corresponding optimal solution to the above SAA problem and $\hat{Z}^{l}$ be the associated optimal inventory investment. Since $Z^{*}$ is always a feasible solution to (6)-(7), we have $\Pi^{N}\left(\hat{Z}^{l}\right) \geq \Pi\left(Z^{*}\right)$ for all $l=1, \ldots, M$. We then have,

$$
E\left[\bar{\Pi}^{N}\right] \geq \Pi\left(Z^{*}\right), \text { where } \bar{\Pi}^{N}=\frac{1}{M} \sum_{l=1}^{M} \Pi^{N}\left(\hat{Z}^{l}\right)
$$

Therefore, $E\left[\bar{\Pi}^{N}\right]$ is used as the estimate of an upper bound of $\Pi\left(Z^{*}\right)$.

In order to have an unbiased estimator of $\Pi\left(\hat{Z}^{l}\right)$, we again generate one large number of independent sample from the demand simulator, $\mathbf{D}^{N^{\prime}}=\left(D^{1}, D^{2}, \ldots, D^{N^{\prime}}\right)$. Then, for each inventory level vector $\hat{Z}^{l}$, compute the estimate of $\Pi\left(\hat{Z}^{l}\right)$ by

$$
\hat{\Pi}^{N^{\prime}}\left(\hat{Z}^{l}\right)=\frac{1}{N^{\prime}} \sum_{h=1}^{N^{\prime}} \pi\left(\hat{Z}^{l}, D^{h}\right)-\sum_{i \in I} c_{i} \hat{z}_{i}^{l}
$$

where $\pi\left(\hat{Z}^{l}, D^{h}\right)$ is the optimal solution to the second stage allocation optimization with inventory vector $\hat{Z}^{l}$ and demand realization $D^{h}$. The estimated optimal inventory vector $\hat{Z}^{*}$ is then determined by choosing the one that gives the largest $\hat{\Pi}^{N^{\prime}}\left(\hat{Z}^{l}\right)$ among all candidate inventory vectors $\hat{Z}^{l}$ with sampled demand realizations, as follows:

$$
\hat{Z}^{*} \in \arg \max \left\{\hat{\Pi}^{N^{\prime}}\left(\hat{Z}^{l}\right), l=1, \ldots, M\right\}
$$

Since $\hat{Z}^{*}$ is a feasible solution to the basic model (1)-(5), we further have

$$
E\left[\hat{\Pi}^{N^{\prime}}\left(\hat{Z}^{*}\right)\right] \leq \Pi\left(Z^{*}\right)
$$

As a result, $E\left[\hat{\Pi}^{N^{\prime}}\left(\hat{Z}^{*}\right)\right]$ can serve as a lower bound of $\Pi\left(Z^{*}\right)$. Thus, the difference between $E\left[\bar{\Pi}^{N}\right]$ and $E\left[\hat{\Pi}^{N^{\prime}}\left(\hat{Z}^{*}\right)\right]$ is an estimate of the optimality gap of SAA solution. In brief, the SAA method works in the following procedure:

Step $0 \quad$ Determine appropriate values for $N, M$ and $N^{\prime}$, and initialize $l=0$;

Step 1 Set $l=l+1$ and generate an independent sample $\mathbf{D}^{l}=\left(D^{l, 1}, D^{l, 2}, \ldots, D^{l, N}\right)$; Solve the SAA problem (6)-(7) for $\hat{Z}^{l}$ and $\Pi^{N}\left(\hat{Z}^{l}\right)$; If $l<M$, go to Step 1; otherwise, go to Step 2;

Step 2 Generate an independent sample $\mathbf{D}^{N^{\prime}}=\left(D^{1}, D^{2}, \ldots, D^{N^{\prime}}\right)$; Initialize $l=0$;

Step 3 Set $l=l+1$ and solve the SAA problem with $\mathbf{D}^{N^{\prime}}$ and $\hat{Z}^{l}$ for $\hat{\Pi}^{N^{\prime}}\left(\hat{Z}^{l}\right)$; If $l<M$, go to Step 2; otherwise go to Step 4;

Step 4 Choose $\hat{Z}^{*} \in \arg \max \left\{\hat{\Pi}^{N^{\prime}}\left(\hat{Z}^{l}\right), l=1, \ldots, M\right\}$;

The quality of the solution, measured by the optimality gap, improves as the sample sizes $N$ and $N^{\prime}$ grow. However, larger sample sizes require higher computational capacity. Therefore, tradeoff between sample sizes and computational effort need to be considered.

\section{NUMERICAL STUDY}

In this section, we apply the proposed approach to a real-world flour manufacturing system with one-year demand data and some scaled cost values. The system produces 18 kinds of flour " $A$ " to " $R$ " for different uses from 3 grades of wheat numbered " 1 " to " 3 " from different origins. The ingredients are mainly starch, protein and fiber. The wheat with higher protein concentration costs more. The costs, ingredient concentration, and requirement matrices are summarized in Table 2. 
He, Huang, and Shen

Table 2: Parameters in Numerical Study

\begin{tabular}{l|ll|l|lll}
\hline & $\begin{array}{l}\text { Raw material cost } \\
(\$ / \mathrm{kg})\end{array}$ & $\begin{array}{l}\text { Grade selection cost } \\
\left(10^{3} \$\right)\end{array}$ & $\begin{array}{l}\text { Price } \\
(\$ / \mathrm{kg})\end{array}$ & $\begin{array}{l}\text { Starch } \\
(100 \%)\end{array}$ & $\begin{array}{l}\text { Protein } \\
(100 \%)\end{array}$ & $\begin{array}{l}\text { Fiber } \\
(100 \%)\end{array}$ \\
\hline Wheat 1 & 2.10 & 2.0 & & 0.80 & 0.10 & 0.10 \\
Wheat 2 & 1.83 & 2.5 & N/A & 0.60 & 0.15 & 0.25 \\
Wheat 3 & 1.78 & 3.0 & & 0.50 & 0.30 & 0.20 \\
\hline Product A & & & 2.63 & 0.98 & 0 & 0.02 \\
Product B & & 2.43 & 0.95 & 0 & 0.05 \\
Product C & & 2.21 & 0.72 & 0.1 & 0.18 \\
Product D & & 2.38 & 0.88 & 0 & 0.12 \\
Product E & & 2.40 & 0.88 & 0.02 & 0.1 \\
Product F & & 2.19 & 0.8 & 0 & 0.2 \\
Product G & & 2.15 & 0.68 & 0.15 & 0.17 \\
Product H & & 2.41 & 0.93 & 0 & 0.07 \\
Product I & & 2.06 & 0.75 & 0 & 0.25 \\
Product J & N/A & & 1.79 & 0.65 & 0 & 0.35 \\
Product K & & 1.77 & 0.2 & 0.7 & 0.1 \\
Product L & & 2.33 & 0.76 & 0.1 & 0.14 \\
Product M & & 2.13 & 0.57 & 0.2 & 0.23 \\
Product N & & 2.37 & 0.57 & 0.33 & 0.1 \\
Product O & & 2.23 & 0.33 & 0.52 & 0.15 \\
Product P & & 2.41 & 0.3 & 0.65 & 0.05 \\
Product Q & & 2.36 & 0.09 & 0.87 & 0.04 \\
Product R & & 1.48 & 0 & 1 & 0 \\
\hline
\end{tabular}

The sample demand arrivals are generated by the demand simulator module, which implements Bootstrap sampling in the current setting. The SAA optimizer module is realized via CPLEX solver with $N=100$, $M=30$ and $N^{\prime}=500$. We compute the average profit, inventory investment in dollar value and gaps for various parameter settings listed in Table 3. As mentioned in the SAA algorithm, the gap is defined as the difference between the upper and lower bounds. The upper bound is estimated by $\bar{\Pi}^{N}=\frac{1}{M} \sum_{l=1}^{M} \Pi^{N}\left(\hat{Z}^{l}\right)$ and the lower bound is estimated by $\frac{1}{N^{\prime}} \sum_{h=1}^{N^{\prime}} \pi\left(\hat{Z}^{*}, D^{h}\right)-\sum_{i \in I} c_{i} \hat{z}_{i}^{*}$. For current parameter setting, the optimal solution given by our approach is $\$ 2721.74 \times 10^{6}$ with inventory levels [5718.194;0;4041.851] for wheat 1,2 and 3 respectively. We summarize the computational results of run 1 to 5 for flexible recipe system in Table 4.

Table 3: Experiment Setting of Selected Runs

\begin{tabular}{c|cc}
\hline & Wheat costs & Grade selection costs \\
\hline Run 1 & {$[1.680 ; 1.464 ; 1.424]$} & {$[2 ; 2.5 ; 3]$} \\
Run 2 & {$[1.890 ; 1.647 ; 1.602]$} & {$[2 ; 2.5 ; 3]$} \\
Run 3 & {$[2.100 ; 1.830 ; 1.780]$} & {$[2 ; 2.5 ; 3]$} \\
Run 4 & {$[2.310 ; 2.013 ; 1.958]$} & {$[2 ; 2.5 ; 3]$} \\
Run 5 & {$[2.520 ; 2.196 ; 2.136]$} & {$[2 ; 2.5 ; 3]$} \\
\hline
\end{tabular}

Table 4: Results for Flexible Recipes

\begin{tabular}{l|ccc}
\hline & Avg. profit $\left(10^{6} \$\right)$ & Inv. $\left(10^{6} \$\right)$ & Gap \\
\hline Run 1 & 6750.69 & 17631.84 & $1.82 \%$ \\
Run 2 & 4666.52 & 18948.49 & $0.91 \%$ \\
Run 3 & 2721.74 & 19275.04 & $0.05 \%$ \\
Run 4 & 938.55 & 18000.56 & $0.41 \%$ \\
Run 5 & 30.22 & 3310.80 & $0.15 \%$ \\
\hline
\end{tabular}

For the selected run 1 to 5, all gaps are less than $2 \%$. This suggests the good performance of the simulation-based approach with our choice of N, M and N'. If the gap is big, the number of samples $\mathrm{N}$ 


\section{He, Huang, and Shen}

and N' should be increased accordingly. Besides, both the average profit and total inventory investment decrease convexly as the raw material cost increases. This implies that the system tends to stock less inventory when raw materials cost is high. Recall that in newsvendor model, the critical fractile decreases linearly with production cost and thus the inventory level also decreases convexly if the demand distribution is concave, i.e. Normal distribution.

The impact of large grade selection cost is illustrated in Figure 5. The increasing grade selection costs decrease the average profit at a mild rate. Meanwhile, grade selection cost increase does not change the inventory investment significantly. Since the grade selection costs are only scaled by multipliers, their relative ranking among different raw materials are not changed. As a result, the preference among wheats are not much affected. This makes the inventory investment decision and average revenue almost unchanged. Therefore, the average profit, which equals average revenue less inventory investment and grade selection costs, decreases linearly in the multipliers of grade selection costs.

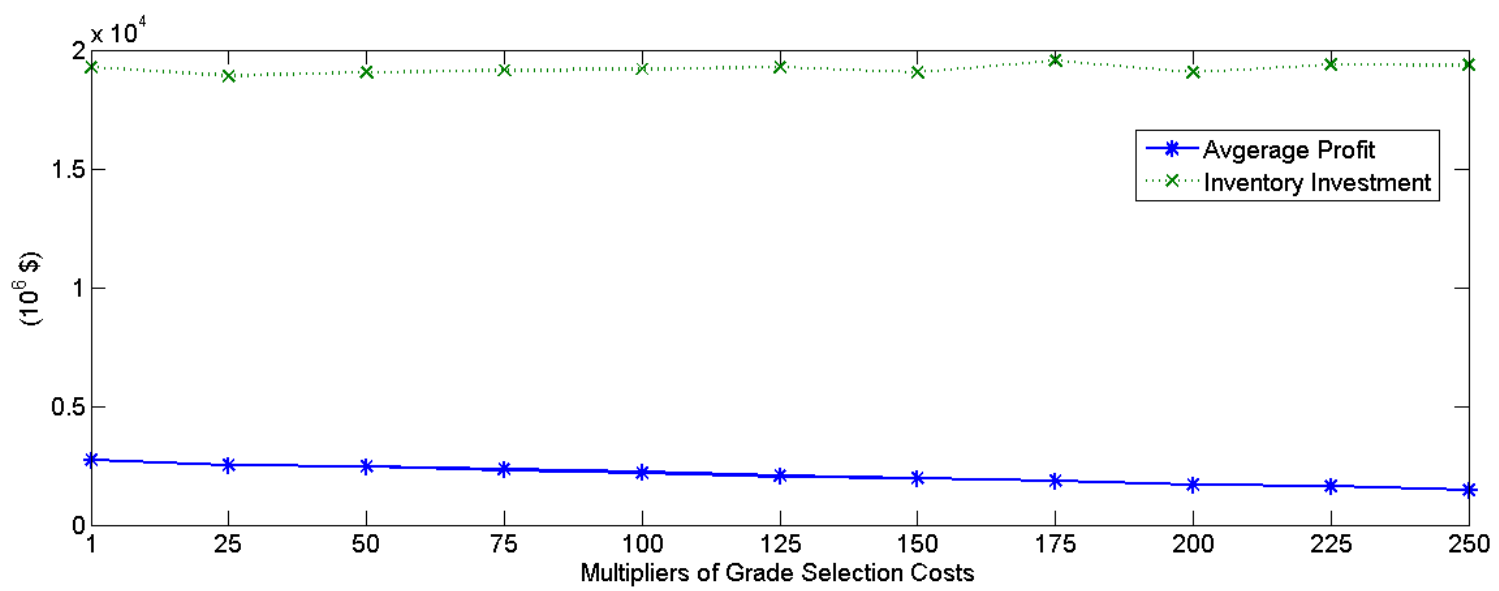

Figure 5: Average Profit and Inventory Investment for Different Grade Selection Costs

We consider three simple fixed recipes. That is, fixed recipes with single source: wheat 1,2 or 3 . Table 5 summarizes the computational results of the average profit, inventory investment as well as the ratio between average profit of the flexible recipes and those of the fixed recipes. For all experiments, flexible recipes can always achieve larger average profit than fixed recipes. For example, in run 1, the "best" fixed recipe can at most generate $86.43 \%$ of the average profit of flexible recipe. Meanwhile, in run 5, which is an extreme case, wheat 1 and 2 are too costly to be used as raw materials. This leaves wheat 3 as the only choice as raw material for the flexible recipe. Therefore, in run 5, the flexible recipe is equivalent to the fixed recipe with wheat 3 . In addition, as we see from run 1 to 3 , the optimal solution of the flexible recipes requires not much more or even significantly less inventory investment than the "best" fixed recipes. That is, flexible recipes achieve higher average profit with lower inventory investment than fixed recipes.

Table 5: Comparison of Flexible Recipes and Fixed Recipes

\begin{tabular}{l|l|l|l|l|l|l|l|l|l|l|l}
\hline & \multicolumn{2}{|c|}{ Flexible recipe } & \multicolumn{3}{c|}{ Fixed recipe: wheat 1 } & \multicolumn{3}{c|}{ Fixed recipe:wheat 2 } & \multicolumn{3}{c}{ Fixed recipe: wheat 3 } \\
\hline & $\begin{array}{l}\text { Avg. } \\
\text { profit } \\
\left(10^{6} \$\right)\end{array}$ & $\begin{array}{l}\text { Inv. } \\
\left(10^{6} \$\right)\end{array}$ & $\begin{array}{l}\text { Avg. } \\
\text { profit } \\
\left(10^{6} \$\right)\end{array}$ & Ratio & $\begin{array}{l}\text { Inv. } \\
\left(10^{6} \$\right)\end{array}$ & $\begin{array}{l}\text { Avg. } \\
\text { profit } \\
\left(10^{6} \$\right)\end{array}$ & Ratio & $\begin{array}{l}\text { Inv. } \\
\left(10^{6} \$\right)\end{array}$ & $\begin{array}{l}\text { Avg. } \\
\text { profit } \\
\left(10^{6} \$\right)\end{array}$ & $\begin{array}{l}\text { Ratio } \\
\text { Inv. } \\
\left(10^{6} \$\right)\end{array}$ \\
\hline Run 1 & 6750.69 & 17631.84 & 4869.70 & $72.14 \%$ & 14188.77 & 5834.94 & $86.43 \%$ & 18140.89 & 5039.26 & $74.65 \%$ & 16165.77 \\
Run 2 & 4666.52 & 18948.49 & 3244.55 & $69.53 \%$ & 14384.60 & 3551.48 & $76.11 \%$ & 18677.88 & 3151.62 & $67.54 \%$ & 14579.06 \\
Run 3 & 2721.74 & 19275.04 & 1695.32 & $62.29 \%$ & 14035.47 & 1595.87 & $58.63 \%$ & 19062.30 & 1786.61 & $65.64 \%$ & 12421.80 \\
Run 4 & 938.55 & 18000.56 & 448.34 & $47.77 \%$ & 11636.23 & 85.50 & $9.11 \%$ & 7330.68 & 695.27 & $74.08 \%$ & 10315.34 \\
Run 5 & 30.22 & 3310.80 & 0 & $0 \%$ & 0 & 0 & $0 \%$ & 0 & 29.62 & $98.02 \%$ & 3256.07 \\
\hline
\end{tabular}




\section{He, Huang, and Shen}

\section{CONCLUSION AND FUTURE WORK}

In this paper, we propose a two-stage stochastic mixed-integer program to an inventory management problem in batch process with flexible recipes. In the first stage, the model determines inventory levels for each period based on past demand data. After demand arrivals are realized, the second stage recourse makes recipe selection and allocation decisions in production. With available historical demand data, a simulation-based approach based on SAA algorithm is developed to solve the stochastic program. As the historical demand data updates along the time, the inventory levels are set iteratively in each period using the most updated demand information. The results of numerical study show the performance of the approach on various cost settings as well as the benefits of flexible recipes over fixed recipes.

In the proposed approach, we focus on the application of the SAA algorithm and use Bootstrap sampling as the default in demand simulation. A direction of future improvement is to incorporate better techniques in the simulation of future demand arrivals based on historical demand data. Those techniques may consider some properties of the demand, such as seasonality and autocorrelation. Also, with limited demand information, a robust optimization model might be developed that considers the worst cases. Moreover, since our model assumes any inventory leftover at the end of each period is disposed, the extension that relaxes this assumption and introduces inventory holding cost in multi-period setting should also be investigated.

\section{ACKNOWLEDGEMENTS}

This research was partially supported by the National Science Foundation Grants CMMI 1068862, CMMI1031637, CMMI1265671 and the National Science Foundation of China Grants 71071084, 71128001, 71210002 .

\section{REFERENCES}

Akçay, Y., and S. Xu. 2004. "Joint inventory replenishment and component allocation optimization in an assemble-to-order system". Management Science 50 (1): 99-116.

Connor, S. J. 1986. "Process Industry Thesaurus". American Production \& Inventory Control Society.

Fine, C., and R. Freund. 1990. "Optimal investment in product-flexible manufacturing capacity". Management Science 36 (4): 449-466.

Harrison, J., and J. Van Mieghem. 1999. "Multi-resource investment strategies: Operational hedging under demand uncertainty". European Journal of Operational Research 113 (1): 17-29.

Karmarkar, U., and K. Rajaram. 2001. "Grade selection and blending to optimize cost and quality". Operations Research 49 (2): 271-280.

Keesman, K. J. 1993. "Application of flexible recipes for model building, batch process optimization and control". AIChE journal 39 (4): 581-588.

Romero, J., A. Espuña, F. Friedler, and L. Puigjaner. 2003. "A new framework for batch process optimization using the flexible recipe". Industrial \& Engineering Chemistry Research 42 (2): 370-379.

Rutten, W., and J. Bertrand. 1998. "Balancing stocks, flexible recipe costs and high service level requirements in a batch process industry: A study of a small scale model". European Journal of Operational Research 110 (3): 626-642.

Schuster, E., and S. Allen. 1998. “Raw Material Management at Welchs, Inc.”. INTERFACES 28 (5): 13-24.

Shapiro, A., and T. Homem-de Mello. 1998. "A simulation-based approach to two-stage stochastic programming with recourse". Mathematical Programming 81 (3): 301-325.

Van Mieghem, J. 1998. "Investment strategies for flexible resources". Management Science 44 (8): 10711078. 


\section{AUTHOR BIOGRAPHIES}

LONG HE received the B.Eng degree in Logistics Management and Engineering from The Hong Kong University of Science and Technology, Hong Kong in 2010. He is currently pursuing the Ph.D. degree in Industrial Engineering and Operations Research from the University of California at Berkeley, Berkeley CA, United States. His research interests includes supply chain management, capacity planning and inventory control. His email address is longhe@berkeley.edu.

SIMIN HUANG is a Professor and Associate Head of the Department of Industrial Engineering, Tsinghua University. He received his Ph.D. from SUNY at Buffalo in 2004. He has served as associate editor of IIE Transactions since 2005, the executive council member of the China Society of Logistics since 2006, editorial board member of Industrial Engineering Journal (Chinese) since 2011. His current research interests include supply chain risk management, scheduling and network design. His email address is huangsimin@mail.tsinghua.edu.cn.

ZUO-JUN MAX SHEN is the Chancellor's Professor in the department of Industrial Engineering and Operations Research at UC Berkeley. He is also affiliated with Tsinghua University. He received his Ph.D. from Northwestern University in 2000. He has been active in the following research areas: integrated supply chain design and management, market mechanism design, applied optimization, and decision making with limited information. He is currently on the editorial/advisory board for several leading journals. He received the CAREER award from National Science Foundation in 2003. His e-mail is shen@ieor.berkeley.edu. 